\title{
Detection and calibration of microdeletions and microduplications by array-based comparative genomic hybridization and its applicability to clinical genetic testing
}

${ }^{\star}$ Andrew Wong, $P h D^{1}$, ${ }^{\star}$ Christa Lese Martin, $P h D^{1}$, Konstantina Heretis, $B S^{1}$, Teresa Ruffalo, $B S^{2}$, Kim Wilber, $B S^{2}$, Walter King, $P h D^{2}$, and David H. Ledbetter, $P h D^{1}$

\begin{abstract}
Purpose: Genome-wide telomere screening by fluorescence in situ hybridization (FISH) has revealed that $\approx 6 \%$ of unexplained mental retardation is due to submicroscopic telomere imbalances. However, the use of FISH for telomere screening is labor intensive and time consuming, given that 41 telomeres are interrogated. We have evaluated the use of array-based Comparative Genomic Hybridization (aCGH) as a more efficient tool for identifying telomere rearrangements. Methods: In this study, 102 individuals with unexplained mental retardation, with either normal or abnormal FISH results, were selected for a blinded retrospective study using aCGH. Results between the two methodologies were compared to ascertain the ability of aCGH to be used in a clinical diagnostics setting. Results: We detected $100 \%$ of all imbalances previously identified by FISH $(n=17)$ and identified two additional abnormalities, a 10q telomere duplication and an interstitial duplication of 22q11. Interphase FISH analysis verified all abnormal array results. We also demonstrated that aCGH can accurately calibrate the size of telomere imbalances by using an array with "molecular rulers" for the telomeric regions of 1p, 16p, 17p, and 22q. Conclusion: This study demonstrates that aCGH is an equivalent methodology to telomere FISH for detecting submicroscopic deletions. In addition, small duplications that are not easily visible by FISH can be accurately detected using aCGH. Because aCGH allows simultaneous interrogation of hundreds to thousands of DNA probes and is more amenable to automation, it offers an efficient and high-throughput alternative for detecting and calibrating unbalanced rearrangements, both of the telomere region, as well as other genomic locations. Genet Med 2005:7(4):264-271.
\end{abstract}

Key Words: array-based CGH, FISH, molecular rulers, telomere, molecular cytogenetics

Over the past several years, there has been a dramatic increase in the use of genome-wide telomere fluorescence in situ hybridization (FISH) analysis for examining individuals with idiopathic mental retardation with or without congenital malformations. These studies have demonstrated that a significant proportion $(\approx 6 \%)$ of this patient cohort has unbalanced abnormalities involving the telomeres of human chromosomes (reviewed in Biesecker ${ }^{1}$ ). Strikingly, 50\% of these abnormal telomere cases have been shown to be familial, which has ob-

\footnotetext{
From the ${ }^{1}$ Department of Human Genetics, Emory University School of Medicine, Atlanta, Georgia; ${ }^{2}$ Abbott Vysis, Inc., Downer's Grove, Illinois.

David H. Ledbetter, PhD, Department of Human Genetics, Emory University School of Medicine, 615 Michael Street, Atlanta, GA 30322.

${ }^{*}$ Both authors contributed equally to this work.

Online Tables 1 and 2 are available via the ArticlePlus feature at the Genetics in Medicine Web site, http://www.geneticsinmedicine.org

Received: October 21, 2004.

Accepted: January 5, 2005.
}

DOI: 10.1097/01.GIM.0000160076.14102.EC vious implications for genetic counseling and recurrence risk estimates. $^{2}$

There is now widespread availability of commercial kits for multiplex telomere FISH screening, which have rapidly become incorporated into the routine clinical evaluation of individuals with idiopathic mental retardation. However, the use of FISH for telomere screening is labor intensive and time consuming, given that 41 unique telomeres are interrogated. Other assays have also been developed for studying telomere integrity, including microsatellite marker analysis, ${ }^{3}$ multiplex amplifiable probe hybridization (MAPH) ${ }^{4}$ and multiplex ligation-dependent probe amplification (MLPA)..$^{5}$ Some of these assays still take a considerable amount of set-up and analysis time, thus they may not be optimal for routine clinical testing. More importantly, many of these techniques use "telomere markers" that are much more proximal than the set of unique telomere probes developed within $\approx 300 \mathrm{~kb}$ of the end of the chromosome. ${ }^{6}$ Pathologic telomere rearrangements have been reported that involve less then $200 \mathrm{~kb}$ of DNA. ${ }^{7}$ Therefore, the use of such assays could lead to missed diagnoses. 
Array-based Comparative Genomic Hybridization ( $a \mathrm{CGH}$ ) is a molecular cytogenetic technique that is used to detect unbalanced chromosomal rearrangements. ${ }^{8}$ In an $a \mathrm{CGH}$ assay, genomic clones are arrayed on slides to which differentially labeled patient and normal reference DNA are cohybridized. The copy number of each clone is determined based on the ratio of the hybridization signal between the patient and normal reference DNA. Multiple studies have demonstrated the ability of $a \mathrm{CGH}$ to successfully detect chromosomal abnormalities $^{9-12}$ and a whole chromosome 22 microarray has been used to distinguish between hemizygous and homozygous deletions. ${ }^{13}$

Veltman et al. ${ }^{14}$ first applied $a \mathrm{CGH}$ for a telomere integrity screen in 20 patients. More recently, Harada et al..$^{15}$ used a similar telomere clone array to screen for subtelomeric rearrangements in 69 patients. Although these studies successfully demonstrated that $a \mathrm{CGH}$ can detect copy number gains or losses for 41 telomere regions, a survey of the copy number of only one clone for each telomere region may not provide enough information to accurately interpret the clinical meaning of the imbalance. For example, while a deletion of the distal $130 \mathrm{~kb}$ of chromosome 22q causes mental retardation and speech delay, ${ }^{7,16}$ a deletion of the terminal $1 \mathrm{Mb}$ of $17 \mathrm{p}$ does not show any phenotypic effect, even though this region is gene rich. ${ }^{17}$ In addition, because it has previously been demonstrated that length polymorphisms occur at various chromosome ends, ${ }^{18-20}$ genes within different telomere regions may be tolerant to dosage imbalance and more than one clone for each telomere region may help to facilitate diagnostic interpretations.

We previously used a "molecular ruler" strategy, consisting of well-characterized clone sets spanning the most distal $5 \mathrm{Mb}$ of $10 \mathrm{q}, 16 \mathrm{p}$, and $17 \mathrm{p}$, to calibrate the size of the imbalance in individuals with telomere rearrangements of these regions. ${ }^{21}$ The use of the "molecular ruler" clone sets in a FISH-based assay allowed us not only to define the size of the rearrangements, but also to aid in the development of genotype/phenotype correlations.

To date, there has not been a large study to compare the clinical sensitivity and specificity between telomere FISH analysis and $a$ CGH analysis. Such a study is necessary to assess the feasibility of adapting $a \mathrm{CGH}$ into the clinical setting. Therefore, we conducted a retrospective comparison of results previously obtained by FISH analysis against those from $a$ CGH. Our laboratory has analyzed over 500 patients with unexplained mental retardation by telomere FISH. Of these, we selected 102 individuals with normal G-banding analysis, of which 85 had normal telomere FISH results and 17 had telomeric imbalances, for a blinded study using $a \mathrm{CGH}$. The samples were analyzed using CGH arrays that contain clones for every telomere, as well as clones for all of the microdeletion syndromes and additional selected loci located across the genome representing each chromosome arm. In addition, molecular rulers for the telomeric regions of $1 p, 16 p, 17 p$, and 22q were incorporated to determine if accurate calibration of telomere rearrangements could be completed using this strategy in an array format.

\section{MATERIALS AND METHODS}

\section{Telomere clones and molecular rulers}

We previously developed a set of unique telomere clones for each human telomere region consisting of $41 \mathrm{BAC}$ or PAC clones that mapped within $\approx 300 \mathrm{~kb}$ from the end of each chromosome. ${ }^{6}$ The total number of clones for the set was 41 , rather than 48 , because no efforts were made to identify clones for the short arms of the acrocentric chromosomes, and clones for the pseudoautosomal regions on the $\mathrm{X}$ and $\mathrm{Y}$ chromosomes are shared. All clones were characterized by their distance from the end of the chromosome and their evidence for telomeric localization, which was determined using a combination of FISH (metaphase, interphase, and fiber), sequence analysis, and RH mapping.

To construct the molecular ruler clone sets for the $1 \mathrm{p}, 16 \mathrm{p}$, $17 p$, and $22 q$ telomere regions, the unique telomere clone served as the starting point, and a contig of clones was selected proximal to this clone, spanning the terminal $1 \mathrm{Mb}$ of each chromosome end. In addition to this $1-\mathrm{Mb}$ contig, a clone was identified every $500 \mathrm{~kb}$ up to $5 \mathrm{Mb}$ from the telomeres of $17 \mathrm{p}$ and $22 \mathrm{q}$, and up to $10 \mathrm{Mb}$ from the telomeres of $1 \mathrm{p}$ and $16 \mathrm{p}$. Clones were first selected based on the publicly available UCSC Genome Browser (http://genome.ucsc.edu/). Additional published maps were then utilized to fully complete each molecular ruler clone set. The map position of the $1 \mathrm{p}$ molecular ruler clone set was based on Chen et al.,22 and the 16p set was based on Daniels et al. ${ }^{23}$ and Doggett et al. ${ }^{24}$ The clones for the $17 \mathrm{p}$ molecular ruler were obtained from a detailed physical map of the Lissencephaly/Miller-Dieker region. ${ }^{17}$ The sequence map for chromosome 22 was used to complete the $22 \mathrm{q}$ molecular ruler. $^{25}$

STS primers were designed based on the sequence of each BAC or PAC clone, and PCR was used for clone identity verification. Metaphase FISH analysis was used to verify the cytogenetic location of each clone and rule out the presence any cross-hybridization. Three-color interphase FISH analysis was used to order the position of the clones by directly labeling clone DNA with Spectrum Orange, Spectrum Green (both from Vysis/Abbott, Inc., Downer's Grove, IL) or diethylaminocoumarin (DEAC, Perkin Elmer, Boston, MA). FISH analysis was performed as previously described. ${ }^{21}$

\section{Array formats}

Two different formats of arrays were used in this study. The first format, the 132-clone telomere array, contains $41 \mathrm{BAC}$ or PAC clones corresponding to each telomere region. ${ }^{6}$ To increase the clinical sensitivity and specificity of the hybridization, a second clone, immediately proximal to the original telomere clone, was included for most telomeres to build redundancy. The second telomere clone was selected using the UCSC Genome Browser and characterized by PCR and FISH analysis in the same manner described earlier for the molecular ruler clone sets. Molecular ruler clone sets from the telomeric regions of chromosomes $1 \mathrm{p}, 16 \mathrm{p}, 17 \mathrm{p}$, and $22 \mathrm{q}$ and multiple 
clones from the $\mathrm{X}$ and $\mathrm{Y}$ chromosomes were also included in this array format (online Table 1).

The second array format utilized in this study, a commercially available 287 clone array (Genosensor Array 300, Abbott Vysis, Inc.), also contains the complete set of telomere clones contained on the telomere array (http://www.vysis.com/ Literature_55760.asp). Additional clones from other clinically significant loci are included on this array as well as clones from additional selected loci across the genome representing each chromosome arm.

\section{Array printing}

The target DNA for printing was prepared according to Snijders et al. ${ }^{26}$ Before array printing, the DNA printing stocks were tested by PCR for the telomere array and PCR and FISH for the GSA 300 to verify their origin. The DNA samples were arrayed in target spots of approximately 75 to $125 \mu \mathrm{m}$ diameter in triplicate on chromium-coated microscope slides using a robotic system (VersArray Chipwriter Pro, BioRad, Hercules, CA).

\section{Patient DNA labeling and hybridization}

Telomere FISH analysis has been performed on over 500 peripheral blood samples from individuals with unexplained mental retardation and a normal G-banding analysis in our laboratory. From this population, following an approved Institutional Review Board protocol, 102 samples, including 85 with normal FISH results and 17 with abnormal results, were selected for a blinded study using $a \mathrm{CGH}$ analysis. To test the ability of the molecular ruler clone sets on the array to accurately calibrate the size of an imbalance, patients with known imbalances of $1 \mathrm{p}, 16 \mathrm{p}$, the Lissencephaly/Miller-Dieker syndrome region on chromosome $17 \mathrm{p},{ }^{17}$ and $22 \mathrm{q}^{7}$ were selectively included in the $a \mathrm{CGH}$ analysis. The FISH results for each sample, except for the sex of the individual, were blinded to the experimenters. For each array experiment, the patient DNA was hybridized against reference DNA of the opposite sex so that the clones from the $\mathrm{X}$ and $\mathrm{Y}$ chromosomes could be used as positive controls for array analysis. For example, $\mathrm{X}$ chromosome clones examined for a test female and reference male sample pair should show a ratio of 2:1 (gain), whereas all $Y$ chromosome clones should show a ratio of 0:1 (loss).

DNA from the patients and normal individuals of the opposite sex (reference DNA) were isolated using a Puregene kit (Gentra System, Inc., Minneapolis, MN) and quantified by fluorometry. Patient genomic DNA of $100 \mathrm{ng}$ was labeled with Cy3-dCTP and an equal amount of normal reference DNA was labeled with Cy5-dCTP using the GSA 300 Microarray Random Priming Labeling kit (Abbott Vysis, Inc.). Briefly, the labeled DNA was digested with $0.15 \mathrm{U}$ of DNase I at $15^{\circ} \mathrm{C}$ for 1 hour. After two rounds of ethanol precipitations, the labeled patient and reference DNA were resuspended and mixed with $25 \mu \mathrm{L}$ hybridization solution (Abbott Vysis, Inc.). After denaturing the mixed labeled DNA at $80^{\circ} \mathrm{C}$ for 10 minutes, the DNAs were preannealed with Cot-1 DNA within the hybridization buffer for 1 hour at $37^{\circ} \mathrm{C}$. For each patient sample, hybridization was set up on either two telomere arrays, one telomere array and one GSA 300 array, or two GSA 300 arrays for 60 to 66 hours at $37^{\circ} \mathrm{C}$. After hybridization, the microarray slides were washed in $1 \mathrm{X} \mathrm{SSC} / 0.1 \% \mathrm{NP}-40$ for 4 minutes at $58^{\circ} \mathrm{C}$ followed by $0.1 \mathrm{X} \mathrm{SSC} / 0.1 \% \mathrm{NP}-40$ for 4 minutes at $58^{\circ} \mathrm{C}$. After a brief $1 \mathrm{X} \mathrm{SSC}$ and $\mathrm{H}_{2} \mathrm{O}$ wash, the slides were mounted in $90 \%$ glycerol and $1 \mathrm{mmol} / \mathrm{L} 4,6$-diamidino-2-phenylindole (DAPI).

\section{Imaging and analysis}

The Cy3, Cy5, and DAPI images for each microarray slide were captured using a CCD camera-based GenoSensor Reader System (Abbott Vysis, Inc.) and analyzed using the associated software. After subtracting the background signal intensity, the software computed the normalized mass ratio mean, defined as the ratio of the sum of the test intensity pixel values to the sum of the reference intensity pixel values. In general, in a patient sample, a ratio of $<0.8$ was interpreted as suggestive of a copy number loss, whereas a ratio $>1.2$ was suggestive of a gain. This threshold for mean ratios was set based on the parameters for conventional CGH analysis.

Several additional criteria were also set to ensure the quality of the data for evaluation. Each clone contained on the microarray was spotted in triplicate to allow a calculation to determine the robustness and reliability of the data. The coefficient of variation $(\mathrm{CV})$, which was used to measure the amount of variation among the triplicate spots of one target clone, is defined as the sample standard deviation divided by the sample mean over the number of contributing spots of the normalized per-spot test to reference ratio. To assess the quality of the hybridization results, we set the acceptable $\mathrm{CV}$ to be equal to or $<6 \%$. In addition, the $P$ value of the null hypothesis that the target has the modal DNA copy number had to be equal to or $<0.01$. Online Table 2 lists the raw data for the mean mass ratio, $\mathrm{CV}$, and $P$ value for each clone in the 18 patients that showed copy number changes in this study (listed in Table 1 ).

Copy number changes that met the criteria for inclusion in array analysis outlined here could still be excluded from the final array analysis if one of the following observations was noted: (1) a target clone showed a copy number change in one hybridization but not in the duplicate hybridization from the same patient; or (2) in a single experiment, which usually involved the simultaneous hybridization of 12 patient DNA samples in duplicate (24 arrays), the change in copy number of the target clone was present in most or all individuals tested. We also excluded clones that consistently gave erratic results $(n=$ 4). These criteria are similar to those outlined in previous $a \mathrm{CGH}$ studies. ${ }^{12,26}$ The telomere FISH results were revealed to the experimenters only after final array results were interpreted.

\section{RESULTS}

\section{Telomere FISH versus aCGH clinical performance}

As shown in Table 1, aCGH detected $100 \%$ of abnormalities identified by telomere FISH analysis. Figure 1 shows represen- 
Table 1

A comparison between $a \mathrm{CGH}$ and telomere FISH results in patients with unbalanced telomere rearrangements

\begin{tabular}{|c|c|c|c|c|}
\hline Patient & $a \mathrm{CGH}$ results & Size estimation by molecular ruler & FISH & $\operatorname{Match}^{a}$ \\
\hline 1 & $1 \mathrm{p}$ tel deletion & $4 \mathrm{Mb}$ & $\operatorname{del}(1 p)$ & Yes \\
\hline 2 & $1 \mathrm{p}$ tel deletion & $6.5 \mathrm{Mb}$ & $\operatorname{del}(1 p)$ & Yes \\
\hline 3 & $3 p$ tel duplication, $9 p$ tel deletion & $\mathrm{ND}^{b}$ & $\operatorname{der}(9) t(3 p ; 9 p)$ & Yes \\
\hline 4 & $4 \mathrm{q}$ tel deletion, $5 \mathrm{p}$ tel duplication & ND & $\operatorname{der}(4) t(4 q ; 5 p)$ & Yes \\
\hline 5 & $6 \mathrm{q}$ tel deletion & ND & $\operatorname{del}(6 q)$ & Yes \\
\hline 6 & $8 \mathrm{p}$ tel deletion & ND & $\operatorname{del}(8 p)$ & Yes \\
\hline 7 & $9 \mathrm{q}$ tel deletion & ND & $\operatorname{del}(9 q)$ & Yes \\
\hline 8 & 10q tel duplication & ND & Normal & No; validated \\
\hline 9 & $12 \mathrm{p}$ tel deletion, $19 \mathrm{q}$ tel duplication & ND & $\operatorname{der}(12) t(12 p ; 19 q)$ & Yes \\
\hline 10 & $16 p$ tel duplication ${ }^{c}$ & $10 \mathrm{Mb}$ & $\operatorname{der}(10) t(10 q ; 16 p)$ & Yes \\
\hline 11 & $16 p$ tel duplication & ND & $\operatorname{der}(10) t(10 q ; 16 p)$ & Yes \\
\hline 12 & $16 \mathrm{p}$ tel duplication ${ }^{d}$ & $4 \mathrm{Mb}$ & $\operatorname{der}(7) t(7 p ; 16 p)$ & Yes \\
\hline 13 & $16 \mathrm{p}$ tel duplication, $17 \mathrm{p}$ deletion ${ }^{e}$ & $3.5 \mathrm{Mb}$ for $16 \mathrm{p}, \approx 2.7 \mathrm{Mb}$ for $17 \mathrm{p}$ & $\operatorname{der}(17) t(16 p ; 17 p)$ & Yes \\
\hline 14 & $17 \mathrm{p}$ tel deletion ${ }^{f}$ & $1.9 \mathrm{Mb}$ & $\operatorname{del}(17 \mathrm{p})$ & Yes \\
\hline 15 & $17 \mathrm{p}$ tel deletion ${ }^{g}$ & ND & $\operatorname{del}(17 p)$ & Yes \\
\hline 16 & $22 \mathrm{q}$ tel deletion ${ }^{h}$ & $130 \mathrm{~kb}$ & $\operatorname{del}(22 q)$ & Yes; validated \\
\hline 17 & $22 \mathrm{q}$ tel deletion & $4 \mathrm{Mb}$ & $\operatorname{del}(22 q)$ & Yes \\
\hline \multirow[t]{2}{*}{18} & $\mathrm{Xq} / \mathrm{Yq}$ tel duplication & ND & $\operatorname{der}(15) t(X / Y q ; 15 p)$ & Yes \\
\hline & 22q11 duplication & ND & Not tested & No; validated \\
\hline
\end{tabular}

${ }^{a}$ Match compares array results with telomere FISH results. For those that did not match, the additional abnormalities detected by array are described.

${ }^{b} \mathrm{ND}$, Not Determined.

'This patient is case 3 in Martin et al. ${ }^{21}$

${ }^{d}$ This patient is case 2 in Martin et al. ${ }^{2}$

${ }^{e}$ This patient is case 1 in Martin et al. ${ }^{21}$

${ }^{f}$ This patient is LP99-086 in Cardoso et al. ${ }^{17}$

${ }^{8}$ This patient is LR01-167 in Cardoso et al. ${ }^{17}$

${ }^{h}$ This patient has been described in Wong et al. ${ }^{7}$

tative ratio images from two cases with unbalanced translocations detected by aCGH, one involving $4 \mathrm{q}$ and $5 \mathrm{p}$, resulting in monosomy for $4 \mathrm{q}$ and trisomy for $5 \mathrm{p}$, and the second between $16 \mathrm{p}$ and $17 \mathrm{p}$, resulting in trisomy for $16 \mathrm{p}$ and monosomy for $17 \mathrm{p}$. Altered ratios for the $\mathrm{X}$ and $\mathrm{Y}$ chromosome clones, resulting from cohybridizing a patient and control of the opposite sex, are also visible in both cases.

aCGH also detected two imbalances that were not identified by telomere FISH analysis. Case 8 showed a gain in copy number of the 10q telomere region for the target clones 66 and 67 (for both targets, mass ratio $=1.44, \mathrm{CV}<2 \%, P<0.001$ ). A representative array image from Case 8 is depicted in Figure $2 \mathrm{~A}$. By metaphase FISH analysis, shown in Figure $2 \mathrm{~B}$, one $10 \mathrm{q}$ homolog showed a slightly brighter signal intensity than that of the other homolog, which was only appreciated upon retrospective review. As illustrated in Figure 2C and 2D, interphase FISH analysis clearly showed a doublet hybridization signal for one 10q homolog, whereas only a single hybridization signal was observed for the other homolog.

In Case 18, as shown in Figure 3A, aCGH detected a duplication of the $\mathrm{Xq} / \mathrm{Yq}$ pseudoautosomal region, which was pre- viously identified by telomere FISH. By telomere FISH analysis and DAPI staining, the Yq heterochromatic region was shown to be translocated to the short arm of chromosome 15, which is a well-described cytogenetic variant without an associated phenotype. However, as depicted in Figure 3A, $a$ CGH also detected a duplication of the clone corresponding to the marker D22S543 near the 22q centromere (GSA 300 target 265, mass ratio $=1.43, \mathrm{CV}=1 \%, P<0.001)$. This locus is not included in the telomere probe set for FISH analysis. As shown in Figures $3 \mathrm{~B}$ through $3 \mathrm{D}$, this finding was validated by metaphase and interphase FISH analysis, respectively.

Overall, we performed $a \mathrm{CGH}$ on 102 patients with unexplained mental retardation, a normal G-banded karyotype, and previous FISH analysis. Approximately $99 \%$ of target clones from both the GSA300 and the telomere array fit the criteria for inclusion in our analyses (described in Materials and Methods). Eighty-four individuals did not show any dosage imbalance, consistent with their normal results from Gbanding and telomere FISH analyses. As shown in Table 1, all previously reported abnormalities by telomere FISH analysis were identified using $a \mathrm{CGH}$ : unbalanced abnormalities were 

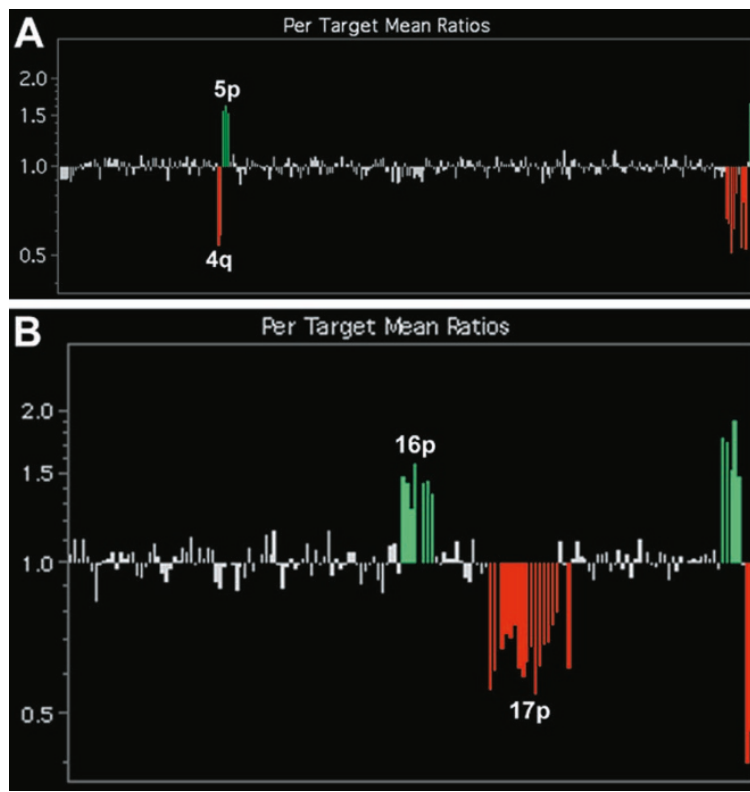

Fig. 1. Representative results from aCGH analysis. A, aCGH results from Case 4, who carries an unbalanced translocation between $4 \mathrm{q}$ and $5 \mathrm{p}$ resulting in monosomy for $4 \mathrm{q}$ (red) and trisomy for $5 \mathrm{p}$ (green). B, $a \mathrm{CGH}$ results for Case 13, who carries an unbalanced translocation between $16 \mathrm{p}$ and $17 \mathrm{p}$ resulting in trisomy for $16 \mathrm{p}$ (green) and monosomy for $17 \mathrm{p}$ (red).
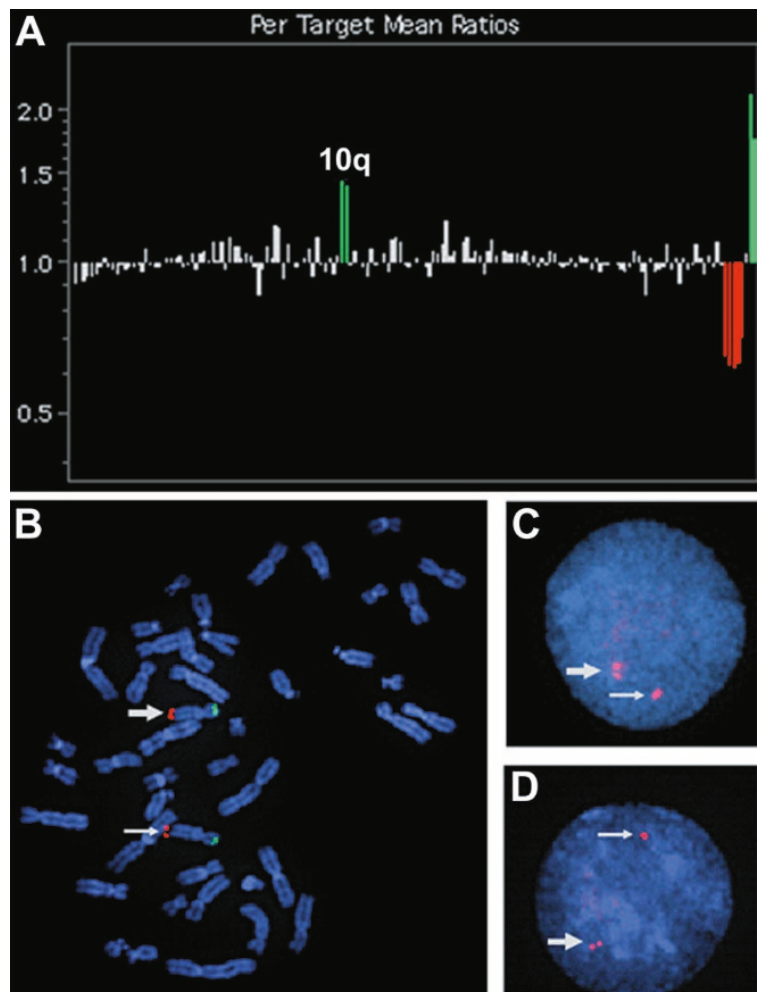

Fig. 2. Microduplication of 10qtel in Case 8. A, aCGH analysis showing a duplication for two 10q clones (green). B, Metaphase image showing hybridization with the $10 \mathrm{p}$ telomere probe (green) and the $10 \mathrm{q}$ telomere (red). The larger arrow indicates the chromosome 10 homolog with a slightly larger hybridization signal; however, no significant difference in signal intensity was observed consistently for the $10 \mathrm{q}$ telomere probe. $\mathrm{C}$ and $\mathrm{D}$, Two interphase nuclei showing one chromosome 10 is duplicated for the $10 \mathrm{q}$ telomere region (indicated by the large arrow and observed as a doublet signal), whereas the normal homolog is single copy (indicated by the small arrow).
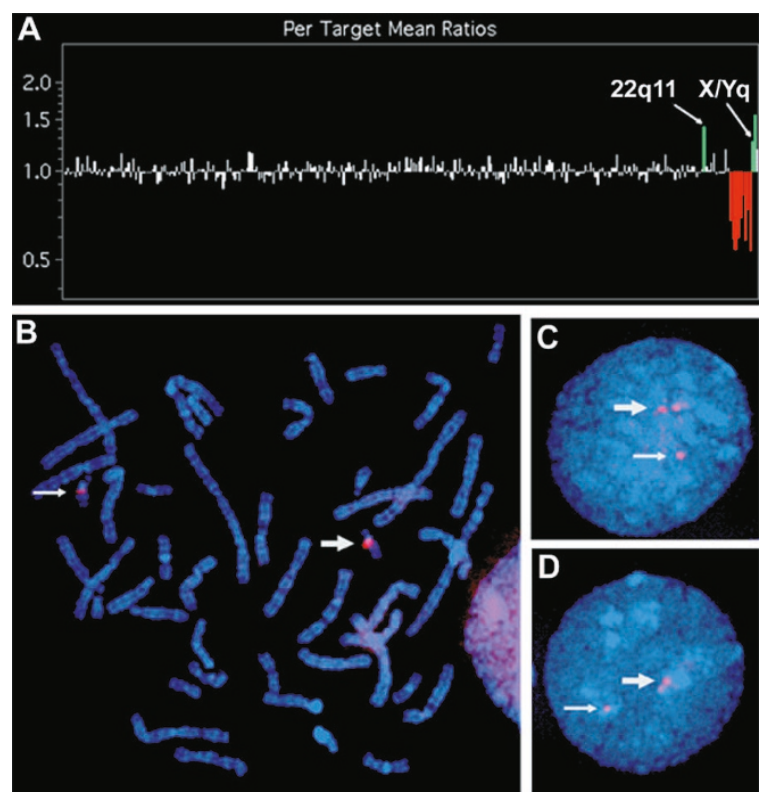

Fig. 3. Interstitial duplication of $22 \mathrm{q} 11$ in Patient 18. A, aCGH analysis showing duplication of X/Yq and 22q11 (green). B, In metaphase chromosomes, one 22q homologue consistently showed a stronger signal using a probe corresponding to D22S543 (indicated by large arrow) than the normal homologue (small arrow). C and D, In two interphase nuclei, duplication of one 22q11 region is shown as a doublet hybridization signal (large arrow), whereas the normal homolog is single copy (small arrow).

identified in 17 individuals including four unbalanced translocations with monosomy and trisomy (Cases $3,4,9$, and 13), 8 terminal deletions (Cases 1, 2, 5, 6, 7, 15, 16, and 17), four duplications (Cases 10,11,12, and 18), and one interstitial deletion (Case 14). As described earlier, two imbalances were detected by aCGH that were not identified by previous G-banding and/or FISH analyses.

As shown in Table 1, we used DNA from 5 individuals whose telomeric deletions or duplications were previously calibrated by FISH (two 16p duplications, ${ }^{21}$ one $17 \mathrm{p}$ deletion, ${ }^{17}$ one $22 \mathrm{q}$ deletion, ${ }^{7}$ and one unbalanced translocation between $16 \mathrm{p}$ and $17 \mathrm{p}^{21}$ ) to evaluate the performance of the molecular ruler clone sets on the telomere array. Figure 1B depicts a representative image from $a \mathrm{CGH}$ analysis for Case 13, an unbalanced translocation between the short arms of chromosomes $16 \mathrm{p}$ and $17 \mathrm{p}$ resulting in trisomy for $16 \mathrm{p}$ and monosomy for $17 \mathrm{p}$. The molecular ruler delineated the size of the $16 \mathrm{p}$ duplication to be $\approx 3.5 \mathrm{Mb}$ (target numbers $81-88$ ) and the size of the $17 \mathrm{p}$ deletion to be $\geq 2.7 \mathrm{Mb}$ (target numbers 102-121).

The estimated size of the imbalance was concordant between the FISH and array analyses for all cases studied. The molecular ruler clone sets were also used for 3 additional cases with telomere deletions of $1 p$ (two cases) and 22q (one case) that were not previously calibrated by FISH. Again, the sizes of the deletions were successfully delineated.

Case 16 carries a $130-\mathrm{kb}$ deletion of the 22qter telomere region, ${ }^{7}$ which is the smallest submicroscopic rearrangement among the patient population examined in this study. One of the target clones (clone 138) on the telomere array spans the breakpoint of this deletion and approximately one fourth of 
the probe covers the nondeleted region. However, this target clone still showed a loss of copy number (mass ratio $=0.72, \mathrm{CV}$ $=1 \%, P<0.001$ ), demonstrating the ability of this technology to identify partial deletions of clones contained on the array.

\section{Polymorphisms}

The target clones corresponding to the telomeric markers 4QTEL11 and 10QTEL24 initially showed ratios suggestive of a duplication in two unrelated samples. These results were validated by follow-up interphase FISH analysis of the probands and subsequent FISH analysis of parental samples showed the duplications to be inherited from a phenotypically normal parent (data not shown). Copy number changes in the clones containing these two markers, 4QTEL11 and 10QTEL24, were later found in $19.6 \%$ and $3 \%$ of the samples, respectively. Therefore, these findings suggest that a DNA sequence length polymorphism is present at these loci and a change in the copy number of these regions most likely represents normal variation among the human population, consistent with recent reports of large scale polymorphisms. ${ }^{27,28}$

\section{DISCUSSION}

$a \mathrm{CGH}$ analysis offers the potential for a higher throughput and less labor intensive approach than FISH analysis to screen for genomic imbalances. To test the feasibility of adopting this technology into a routine clinical cytogenetics setting, we selected 102 patients with unexplained mental retardation and a normal G-banded karyotype who also had previous telomere FISH analysis for $a \mathrm{CGH}$ analysis. Because $a \mathrm{CGH}$ identified all 17 submicroscopic rearrangements that were previously identified by FISH, the clinical sensitivity in this study was $100 \%$.

Interestingly, we uncovered two duplications that were not previously detected by FISH analysis, making $a$ CGH a potentially more sensitive assay for identifying submicroscopic rearrangements. For Case 18, a duplication of the Xq/Yq pseudoautosomal region to the short arm of chromosome 15, which was previously identified by FISH, was also identified by $a$ CGH. This rearrangement is a well-described variant observed by routine cytogenetic analysis. However, $a$ CGH also detected a duplication of the proximal region of the long arm of chromosome 22. The phenotype of this patient included bilateral colobomas, left ptosis, seizures, and developmental delay, which partially overlaps with the clinical features of Cat Eye syndrome. Parental follow-up was not possible for this case. However, because Cat Eye syndrome is caused by a duplication of the proximal region of 22q11, which is usually observed as an additional marker chromosome, ${ }^{29,30}$ but can also be seen as an interstitial duplication, ${ }^{31-33}$ the $22 \mathrm{q} 11$ duplication detected by the array is likely to be related to this individual's phenotype. It also demonstrates that a microarray containing all clinically important target loci is useful for postnatal screening assays.

In Case 8 , a duplication of two clones from the 10q telomere region was identified, which was different from the common polymorphism that we observed involving only one clone from the $10 \mathrm{q}$ region (10QTEL24). In addition to mental retardation, this individual had microcephaly and autism. The duplication was verified in this individual using interphase FISH analysis, and subsequent interphase FISH studies of parental samples demonstrated that the 10q duplication was inherited from his phenotypically normal mother (data not shown). Therefore, it is unlikely that the duplication is related to the phenotypic features observed in the patient. However, this case still demonstrates the ability of $a \mathrm{CGH}$ to identify small duplications that are not readily visible by metaphase FISH analysis.

We previously used FISH analysis with the molecular ruler strategy to estimate the deletion or duplication sizes involved in submicroscopic telomeric rearrangements. ${ }^{17,21}$ As shown in Table 1, when this strategy was applied in an aCGH setting for 8 patients, not only were the imbalances identified correctly, but the sizes of the rearrangements were also determined in a single hybridization. These data demonstrate the power of using a high throughput approach, such as $a \mathrm{CGH}$, in contrast to a FISH approach that would require multiple FISH hybridizations per individual. With the immediate determination of the size of the imbalance available for correlation to the transcriptional map for a particular telomere or genomic region, better interpretations can be made in regards to the clinical significance of the results.

$a \mathrm{CGH}$ has been proven to be accurate for identifying dosage changes when the imbalance occurs in $100 \%$ of a cell population and $100 \%$ of the target clone is either duplicated or deleted. ${ }^{14,26,34}$ There is less data available to establish whether $a \mathrm{CGH}$ can still detect an imbalance if it is mosaic within a cell population or if the target clone is only partially deleted or duplicated. In a study of an $18 \mathrm{q}$ deletion using $a \mathrm{CGH}$, Veltman et al. ${ }^{11}$ demonstrated that if a deletion occurs in $70 \%$ of a cell population, 35 out of 45 target clones showed a mass ratio below 0.8 . The number of target clones that fall below the cutoff ratio was much lower (5 out of 38) if the deletion occurred in only $33 \%$ of the population. Schaeffer et al. ${ }^{35}$ also demonstrated the ability to pick up mosaic cell populations in a study using $a \mathrm{CGH}$ to examine product of conception samples. In a study using an array containing clones for the $\mathrm{X}$ chromosome, Veltman et al. ${ }^{36}$ showed that only half of a clone needs to be deleted in order to be detected by $a \mathrm{CGH}$. In this study, we also demonstrated that for a deletion that covers only $\approx 75 \%$ of a target clone, the mass ratio for that clone was still below 0.8 . Therefore, $a$ CGH can still show a copy number change when the majority of a cell population contains the imbalance or when only a proportion of the target clone is contained within the deleted region.

With the recent completion of the Human Genome Project, ${ }^{37}$ a wealth of genomic resources are readily available. Thus, it is now possible to construct CGH arrays to detect chromosomal abnormalities at a higher resolution than previously possible. To achieve the resolution of a minimal tiling path for each chromosome, an array that covers the whole human genome was constructed based on a human BAC finger-printing map. ${ }^{38}$ Other groups have developed arrays with $\approx 1 \mathrm{Mb}$ resolution. ${ }^{12,26,39,40}$ These "whole genome arrays" are 
definitely a powerful discovery tool for identifying "candidate gene" regions involved in mental retardation and other genetic diseases. However, although a high-density array is invaluable for research, it may not be practical for clinical applications at this time until further studies are performed to identify polymorphisms and assess individual clone performance.

Our $a \mathrm{CGH}$ analysis on a large patient cohort demonstrates that even for a low-density genomic array, we occasionally had to exclude targets from analysis due to the high variation of hybridization signals among triplicate spots. In addition, we have verified by FISH analysis that copy number changes are frequently found in the telomeric regions of $4 \mathrm{q}$ and $10 \mathrm{q}$, containing the markers 4QTEL11 and 10QTEL24, most likely due to sequence-length polymorphisms. As higher-density arrays are utilized, it is extremely likely that more targets across the genome will show changes in copy number that are unrelated to the phenotype of patients and presumably a result of length polymorphisms within the target clones. Recently, two studies have demonstrated that such large scale polymorphisms $(>100 \mathrm{~kb})$ exist in the normal human population..$^{27,28}$

These issues, which are inherent to array analysis, would be magnified as the number of target clones on an array is increased. Therefore, at this time, in order to achieve the highest clinical sensitivity and specificity, a diagnostic array should have selected clone coverage for clinically significant regions. Each target clone should be rigorously screened to be free of low copy repeats that may be polymorphic and could affect the performance of the hybridization. Furthermore, clone redundancy for clinically significant regions is important to increase the likelihood that an imbalance will be identified.

Follow-up FISH confirmation is also imperative for any imbalances identified by $a \mathrm{CGH}$ not only to verify the imbalance, but also to determine the mechanism for the rearrangement. For example, although $a$ CGH identified a duplication in Cases $10,11,12$, and 18 , only metaphase FISH analysis demonstrated that the duplications were the result of an unbalanced translocation where there was no corresponding monosomy identified. For Cases 10, 11, and 12, the breakpoint on the derivative chromosome presumably lies distal to the unique telomere clone, within the repetitive subtelomeric region. Therefore, any resulting monosomy would most likely not be associated with a clinical phenotype, although if one of the parents carries the balanced form of the rearrangement, it would have important implications for recurrence risk estimates and family counseling. Likewise, for Case 18, where the duplicated region is present on the short arm of an acrocentric chromosome, the monosomy for the acrocentric $\mathrm{p}$ arm would not be expected to cause a phenotype, because these regions contain the ribosomal RNA genes that can be lost without phenotypic consequences. However, segregation of the balanced form of this translocation could put other family members at risk for having unbalanced offspring.

Parental FISH analysis is also necessary in determining the clinical significance of any abnormalities identified by $a$ CGH. As demonstrated by Case 8 , a duplication of the $10 \mathrm{q}$ telomere region was identified in an affected proband and his phenotyp- ically normal mother. The lack of phenotypic effect may be related to the involvement of regions of the genome that are tolerant to dosage imbalances. However, there may be as of yet unexplained differences between the parent and child, such as modifier genes or mutations on the nondeleted homolog that are causing the different phenotypes.

Further studies are needed to prospectively evaluate CGH array analysis against currently available diagnostic tests, such as G-banding and FISH. Side-by-side comparisons to accurately estimate the sensitivity and specificity, cost differences, and technician time will aid in determining how array analysis will best be incorporated within routine diagnostic genetic testing.

\section{ACKNOWLEDGMENTS}

This work was supported by funds from Abbott Vysis, Inc. and NIH grant 5 R01 HD36715-03 (to D.H.L.). D.H.L. and C.L.M. have consulting agreements with Vysis, Inc. in the area of molecular cytogenetics. We thank David Johnson, Devan Pressley, June Chung, and Yesim Ilkin for technical support.

\section{References}

1. Biesecker LG. The end of the beginning of chromosome ends. Am J Med Genet 2002;107:263-266.

2. Knight SJ, Regan R, Nicod A et al. Subtle chromosomal rearrangements in children with unexplained mental retardation. Lancet 1999;354:1676-1681.

3. Slavotinek A, Rosenberg M, Knight S et al. Screening for submicroscopic chromosome rearrangements in children with idiopathic mental retardation using microsatellite markers for the chromosome telomeres. J Med Genet 1999;36:405-411.

4. Sismani C, Armour JA, Flint J, Girgalli C, Regan R, Patsalis PC. Screening for subtelomeric chromosome abnormalities in children with idiopathic mental retardation using multiprobe telomeric FISH and the new MAPH telomeric assay. Eur J Hum Genet 2001;9:527-532.

5. Rooms L, Reyniers E, van Luijk R et al. Subtelomeric deletions detected in patients with idiopathic mental retardation using multiplex ligation-dependent probe amplification (MLPA). Hum Mutat 2004;23:17-21.

6. Knight SJ, Lese CM, Precht KS et al. An optimized set of human telomere clones for studying telomere integrity and architecture. Am J Hum Genet 2000;67:320-332.

7. Wong AC, Ning Y, Flint J, et al. Molecular characterization of a 130-kb terminal microdeletion at $22 \mathrm{q}$ in a child with mild mental retardation. Am J Hum Genet 1997;60:113-120.

8. Pinkel D, Segraves R, Sudar D et al. High resolution analysis of DNA copy number variation using comparative genomic hybridization to microarrays. Nat Genet 1998; 20:207-211.

9. Gunn SR, Mohammed M, Reveles XT et al. Molecular characterization of a patient with central nervous system dysmyelination and cryptic unbalanced translocation between chromosomes 4q and 18q. Am J Med Genet 2003;120A:127-135.

10. Ki A, Rauen KA, Black LD et al. Ring 21 chromosome and a satellited $1 \mathrm{p}$ in the same patient: novel origin for an ectopic NOR. Am J Med Genet 2003;120A:365-369.

11. Veltman JA, Jonkers Y, Nuijten I et al. Definition of a Critical Region on Chromosome 18 for Congenital Aural Atresia by ArrayCGH. Am J Hum Genet 2003;72: $1578-1584$.

12. Vissers LE, de Vries BB, Osoegawa K et al. Array-based comparative genomic hybridization for the genomewide detection of submicroscopic chromosomal abnormalities. Am J Hum Genet 2003;73:1261-1270.

13. Buckley PG, Mantripragada KK, Benetkiewicz M et al. A full-coverage, high-resolution human chromosome 22 genomic microarray for clinical and research applications. Hum Mol Genet 2002;11:3221-3229.

14. Veltman JA, Schoenmakers EF, Eussen BH et al. High-throughput analysis of subtelomeric chromosome rearrangements by use of array-based comparative genomic hybridization. Am J Hum Genet 2002;70:1269-1276.

15. Harada N, Hatchwell E, Okamoto N et al. Subtelomere specific microarray based comparative genomic hybridisation: a rapid detection system for cryptic rearrangements in idiopathic mental retardation. J Med Genet 2004;41:130-136.

16. Wilson HL, Wong AC, Shaw SR et al. Molecular characterisation of the 22q13 deletion syndrome supports the role of haploinsufficiency of SHANK3/PROSAP2 in the major neurological symptoms. J Med Genet 2003;40:575-584. 
17. Cardoso C, Leventer RJ, Ward HL et al. Refinement of a 400-kb critical region allows genotypic differentiation between isolated lissencephaly, Miller-Dieker syndrome, and other phenotypes secondary to deletions of 17p13.3. Am J Hum Genet 2003;72:918-930.

18. Macina RA, Negorev DG, Spais C, Ruthig LA, Hu XL, Riethman HC. Sequence organization of the human chromosome 2q telomere. Hum Mol Genet 1994;3:18471853.

19. van Geel M, Dickson MC, Beck AF et al. Genomic analysis of human chromosome $10 \mathrm{q}$ and $4 \mathrm{q}$ telomeres suggests a common origin. Genomics 2002;79:210-217.

20. Wilkie AO, Higgs DR, Rack KA et al. Stable length polymorphism of up to $260 \mathrm{~kb}$ at the tip of the short arm of human chromosome 16. Cell 1991;64:595-606.

21. Martin CL, Waggoner DJ, Wong A et al. "Molecular rulers" for calibrating phenotypic effects of telomere imbalance. J Med Genet 2002;39:734-740.

22. Chen YZ, Hayashi Y, Wu JG et al. A BAC-based STS-content map spanning a 35-Mb region of human chromosome 1p35-p36. Genomics 2001;74:55-70.

23. Daniels RJ, Peden JF, Lloyd C et al. Sequence, structure and pathology of the fully annotated terminal $2 \mathrm{Mb}$ of the short arm of human chromosome 16. Hum Mol Genet 2001;10:339-352.

24. Doggett NA, Goodwin LA, Tesmer JG et al. An integrated physical map of human chromosome 16. Nature 1995;377:335-365.

25. Dunham I, Shimizu N, Roe B et al. The DNA sequence of human chromosome 22. Nature 1999;402:489-495.

26. Snijders AM, Nowak N, Segraves R, et al. Assembly of microarrays for genome-wide measurement of DNA copy number. Nat Genet 2001;29:263-264.

27. Iafrate AJ, Feuk L, Rivera MN, et al. Detection of large-scale variation in the human genome. Nat Genet 2004;36:949-951.

28. Sebat J, Lakshmi B, Troge J et al. Large-scale copy number polymorphism in the human genome. Science 2004;305:525-528.

29. McDermid HE, Duncan AM, Brasch KR et al. Characterization of the supernumerary chromosome in cat eye syndrome. Science 1986;232:646-648.

30. McTaggart KE, Budarf ML, Driscoll DA, Emanuel BS, Ferreira P, McDermid HE. Cat eye syndrome chromosome breakpoint clustering: identification of two inter- vals also associated with 22q11 deletion syndrome breakpoints. Cytogenet Cell Genet 1998;81:222-228.

31. Lindsay EA, Shaffer LG, Carrozzo R, Greenberg F, Baldini A. De novo tandem duplication of chromosome segment 22q11-q12: clinical, cytogenetic, and molecular characterization. Am J Med Genet 1995;56:296-299.

32. Knoll JH, Asamoah A, Pletcher BA, Wagstaff J. Interstitial duplication of proximal 22q: phenotypic overlap with cat eye syndrome. Am J Med Genet 1995;55:221-224.

33. Reiss JA, Weleber RG, Brown MG, Bangs CD, Lovrien EW, Magenis RE. Tandem duplication of proximal 22q: a cause of cat-eye syndrome. Am J Med Genet 1985;20: 165-171.

34. Yu W, Ballif BC, Kashork CD, et al. Development of a comparative genomic hybridization microarray and demonstration of its utility with 25 well-characterized 1 p 36 deletions. Hum Mol Genet 2003;12:2145-2152.

35. Schaeffer AJ, Chung J, Heretis K, Wong A, Ledbetter DH, Lese Martin C. Comparative genomic hybridization-array analysis enhances the detection of aneuploidies and submicroscopic imbalances in spontaneous miscarriages. Am J Hum Genet 2004;74:1168-1174.

36. Veltman JA, Yntema HG, Lugtenberg D et al. High resolution profiling of X chromosomal aberrations by array comparative genomic hybridisation. J Med Genet 2004;41:425-432.

37. Collins FS, Green ED, Guttmacher AE, Guyer MS. A vision for the future of genomics research. Nature 2003;422:835-847.

38. Ishkanian AS, Malloff CA, Watson SK, et al. A tiling resolution DNA microarray with complete coverage of the human genome. Nat Genet 2004;36:299-303.

39. Smirnov DA, Burdick JT, Morley M, Cheung VG. Method for manufacturing whole-genome microarrays by rolling circle amplification. Genes Chromosomes Cancer 2004;40:72-77.

40. Greshock J, Naylor TL, Margolin A et al. 1-Mb resolution array-based comparative genomic hybridization using a BAC clone set optimized for cancer gene analysis. Genome Res 2004;14:179-187. 